\title{
ON THE STRUCTURE OF GABOR AND SUPER GABOR SPACES
}

\author{
LUIS DANIEL ABREU
}

\begin{abstract}
We study the structure of Gabor and super Gabor spaces as subspaces of $L^{2}\left(\mathbb{R}^{2 d}\right)$ and specialize the results to the case where the spaces are generated by vectors of Hermite functions. We then show that such spaces are isometrically isomorphic to Fock spaces of polyanalytic functions and obtain structure theorems and orthogonal projections for both spaces at once. In particular we recover a structure result obtained by N. Vasilevskii using complex analysis and special functions. In contrast, our methods use only time-frequency analysis, exploring a link between time-frequency analysis and the theory of polyanalytic functions, provided by the polyanalytic part of the Gabor transform with a Hermite window, the polyanalytic Bargmann transform.
\end{abstract}

KEYWORDS: time-frequency analysis, Gabor transform and super Gabor transform, Bargmann transform, polyanalytic Fock spaces .

\section{Introduction}

In [1] we have proved sharp results concerning sampling and interpolation in polyanalytic Fock spaces, by relating the problem to vector valued (super) Gabor frames and Riesz sequences with Hermite windows and applying duality principles from time-frequency analysis. Motivated by the connection between Gabor analysis and complex analysis of polyanalytic functions, we have also introduced a vector valued version of the Gabor transform, a map

$$
\mathbf{V}_{\mathbf{g}}: L^{2}\left(\mathbb{R}^{d}, \mathbb{C}^{n}\right) \rightarrow L^{2}\left(\mathbb{R}^{2 d}\right),
$$

which provides the continuous counterpart of the theory of super Gabor frames presented in [3] and developed in the case of Hermite functions in [8], [9] and [6]. Such transform reduces to the Gabor transform when $n=1$.

In this paper we will study the structure of the subspaces of $L^{2}\left(\mathbb{R}^{2 d}\right)$ constituted by the images of $\mathcal{H}=L^{2}\left(\mathbb{R}^{d}, \mathbb{C}^{n}\right)$ under $\mathbf{V}_{\mathbf{g}}$ (Gabor super spaces). We will find analogues of recent results obtained by Hutnik [10] in the context of the scalar wavelet transform. Our first result provides a unitary mapping

$$
U: L^{2}\left(\mathbb{R}^{2 d}\right) \rightarrow \mathcal{H} \otimes \mathcal{H}
$$

Received March 12, 2009.

Partial financial assistance by FCT grant SFRH/BPD/26078/2005 and CMUC. 
such that

$$
U\left(\mathbf{V}_{\mathbf{g}} \mathbf{f}\right)=\langle\mathbf{f}, \mathbf{g}\rangle_{\mathcal{H}} .
$$

Then we will compute the reproducing kernel of the Gabor superspaces by relating them to the scalar case.

By specializing our results to the case where the window $\mathbf{g}$ is constituted by the first $n-1$ Hermite functions, we obtain explicit results for the reproducing kernel (here our approach diverges from that in [10], [11], since we use more direct methods).

Finally, we show that the multiplier

$$
M(z)=e^{\pi \frac{|z|^{2}}{2}-i \pi x \omega}
$$

provides an isometric isomorphism between the Gabor spaces with Hermite windows and the Fock spaces of polyanalytic functions, using some of the results in [1]. This provides a method for the study of polyanalytic Fock spaces by means of time-frequency analysis techniques. Using this method, we recover a result of Vasilevskii [13] concerning the structure of polyanalytic Fock spaces. Moreover, we obtain an explicit formula for the reproducing kernel of the polyanalytic Fock space from where growth estimates can be derived.

The organization of the paper is as follows. We have a background section with the essential tools: the Gabor transform, the Bargmann transform and the Hermite functions. Then, third section is devoted to the study of Gabor and super Gabor spaces with general windows. We then specialize the windows to be Hermite functions and we find a bit more of structure, namely we are able to compute an explicit form of the reproducing kernel. In the last section the polyanalytic Fock spaces are introduced. Combining the results of the previous sections with some results obtained in [1], we obtain the results for the polyanalytic Fock spaces.

\section{Background}

2.1. The Gabor transform. Fix a function $g \neq 0$. Then the Gabor (shorttime) Fourier transform of a function $f$ with respect to the "window" $g$ is defined, for every $x, \omega \in \mathbb{R}^{d}$ as

$$
V_{g} f(x, \omega)=2^{\frac{d}{4}} \int_{\mathbb{R}^{d}} f(t) \overline{g(t-x)} e^{-2 \pi i t \omega} d t .
$$


The Gabor transform provides an isometry

$$
V_{g}: L^{2}\left(\mathbb{R}^{d}\right) \rightarrow L^{2}\left(\mathbb{R}^{2 d}\right),
$$

that is, if $f, g \in L^{2}\left(\mathbb{R}^{d}\right)$, then

$$
\left\|V_{g} f\right\|_{L^{2}\left(\mathbb{R}^{2 d}\right)}=\|f\|_{L^{2}\left(\mathbb{R}^{d}\right)}\|g\|_{L^{2}\left(\mathbb{R}^{d}\right)} .
$$

The following relations are usually called the orthogonal relations for the short-time Fourier transform. Let $f_{1}, f_{2}, g_{1}, g_{2} \in L^{2}\left(\mathbb{R}^{d}\right)$. Then $V_{g_{1}} f_{1}, V_{g_{2}} f_{2} \in$ $L^{2}\left(\mathbb{R}^{2 d}\right)$ and

$$
\left\langle V_{g_{1}} f_{1}, V_{g_{2}} f_{2}\right\rangle_{L^{2}\left(\mathbb{R}^{2 d}\right)}=\left\langle f_{1}, f_{2}\right\rangle_{L^{2}\left(\mathbb{R}^{d}\right)} \overline{\left\langle g_{1}, g_{2}\right\rangle_{L^{2}\left(\mathbb{R}^{d}\right)}} .
$$

For every $x, \omega \in \mathbb{R}^{d}$ define the operators translation by $x$ and modulation by $\omega$ as

$$
\begin{aligned}
T_{x} f(t) & =f(t-x), \\
M_{\omega} f(t) & =e^{2 \pi i \omega t} f(t) .
\end{aligned}
$$

Using these operators we can write (1) as

$$
V_{g} f(x, \omega)=\left\langle f, M_{\omega} T_{x} g\right\rangle_{L^{2}\left(\mathbb{R}^{d}\right)} .
$$

Using the Fourier transform

$$
\mathcal{F} f(\omega)=\int_{\mathbb{R}^{d}} f(x) e^{-2 \pi i x \omega} d x,
$$

the operators translation by $x$ and modulation by $\omega$ can be related in the following way:

$$
T_{x} f=\mathcal{F}^{-1}\left(M_{-x}(\mathcal{F} f)\right) .
$$

2.2. The Bargmann transform. Here we will use multi-index notation, $z=\left(z_{1}, \ldots z_{d}\right), n=\left(n_{1, \ldots} n_{d}\right)$ and $|n|=n_{1}+\ldots+n_{d}$. The Bargmann transform is defined by

$$
(B f)(z)=2^{\frac{d}{4}} \int_{\mathbb{R}^{d}} f(t) e^{2 \pi t z-\pi z^{2}-\frac{\pi}{2} t^{2}} d t
$$

It is an isomorphism

$$
B: L^{2}\left(\mathbb{R}^{d}\right) \rightarrow \mathcal{F}\left(\mathbb{C}^{d}\right)
$$

where $\mathcal{F}\left(\mathbb{C}^{d}\right)$ stands for the Bargmann-Fock space of analytic functions in $\mathbb{C}^{d}$ with the norm

$$
\|F\|_{L^{2}\left(\mathbb{C}^{d}, e^{\left.-\pi|z|^{2}\right)}\right.}^{2}=\int_{\mathbb{C}^{d}}|F(z)|^{2} e^{-\pi|z|^{2}} d z .
$$


The collection of the monomials of the form

$$
e_{n}(z)=\left(\frac{\pi^{|n|}}{n !}\right)^{\frac{1}{2}} z^{n}=\prod_{j=1}^{d} \frac{\pi^{n_{j}}}{\sqrt{n_{j} !}} z^{n_{j}}, n=\left(n_{1, \ldots} n_{d}\right)
$$

with $n_{i} \geq 0$, constitutes an orthonormal basis of $\mathcal{F}\left(\mathbb{C}^{d}\right)$. The reproducing kernel of $\mathcal{F}\left(\mathbb{C}^{d}\right)$ is the function

$$
K^{(0)}(z, w)=e^{\pi \bar{w} z}
$$

A simple calculation shows that the Bargmann transform is related to the Gabor transform with the Gaussian window $\varphi(x)=2^{\frac{d}{4}} e^{-\pi x^{2}}$ by the formula

$$
V_{\varphi} f(x,-\omega)=e^{i \pi x \omega} e^{-\pi \frac{|z|^{2}}{2}}(B f)(z),
$$

where $z=x+i \omega$.

2.3. The Hermite functions. The Hermite functions can be defined via the so called Rodrigues Formula

$$
h_{n}(t)=c_{n} e^{\pi t^{2}}\left(\frac{d}{d t}\right)^{n}\left(e^{-2 \pi t^{2}}\right) .
$$

where $c_{n}$ is choosen in such a way they can provide an orthonormal basis of $L^{2}(\mathbb{R})$. Now let $n=\left(n_{1, \ldots} n_{d}\right)$ and $x \in \mathbb{R}^{d}$. The $d$-dimensional Hermite functions are

$$
\Phi_{n}(x)=\prod_{j=1}^{n} h_{n_{j}}(x) .
$$

They form a complete orthonormal system of $L^{2}\left(\mathbb{R}^{d}\right)$.

A very important property of the Hermite functions is that they are mapped into a basis of the Bargmann-Fock space via the Bargmann transform:

$$
\left(B \Phi_{n}\right)(z)=e_{n}(z)
$$

\section{Gabor spaces and Gabor super spaces}

3.1. Gabor spaces. Let $\mathcal{G}_{g}$ denote the subspace of $L^{2}\left(\mathbb{R}^{2 d}\right)$ which is the image of $L^{2}\left(\mathbb{R}^{d}\right)$ under the Gabor transform with the window $g$,

$$
\mathcal{G}_{g}=\left\{V_{g} f: f \in L^{2}\left(\mathbb{R}^{d}\right)\right\} .
$$


The spaces $\mathcal{G}_{g}$ are called "model spaces" in [2]. It is well known (see [5]) that Gabor spaces have a reproducing kernel given by

$$
k(x, \omega, u, \eta)=\left\langle M_{\omega} T_{x} g, M_{\eta} T_{u} g\right\rangle_{L^{2}\left(\mathbb{R}^{d}\right)}
$$

For instance, if we consider the Gaussian window $\varphi(x)=2^{\frac{d}{4}} e^{-\pi x^{2}}$, a calculation (see [7, Lemma 1.5.2]) shows that the reproducing kernel of $\mathcal{G}_{g}$ is

$$
k^{0}(x, \omega, u, \eta)=e^{\pi i(u+x)(\omega-\eta)-\frac{\pi(u-x)^{2}-\pi(\eta-\omega)^{2}}{2}} .
$$

Using the notation $z=x+i \omega$ and $w=u+i \eta$, this reproducing kernel can be related with the reproducing kernel (7) of the Fock space in the following way:

$$
k^{0}(z, w)=e^{-i \pi(u \eta-x \omega)-\pi \frac{|z|^{2}+|w|^{2}}{2}} e^{\pi \bar{w} z} .
$$

In the remaining of the paper we will use repeatedly the following fact, valid in any space $H$ with a reproducing kernel $K(z, w)$ : if we can estimate the diagonal function $K(w, w)$, we automatically have estimates for the growth of an arbitrary function $F \in H$, since

$$
|F|=\left|\langle F, K(., w)\rangle_{H}\right| \leq\|F\|_{H}\|K(., w)\|_{H}=\|F\|_{H} \sqrt{K(w, w)} .
$$

As a simple example, observe that $k^{(0)}(z, z)=1$. As a result, elements in $\mathcal{G}_{g}$ satisfy $|F| \leq\|F\|_{G_{g}}$.

3.2. Super Gabor spaces. Consider the Hilbert space $\mathcal{H}=L^{2}\left(\mathbb{R}^{d}, \mathbb{C}^{n}\right)$, consisting of vector-valued functions $\mathbf{f}=\left(f_{0}, \ldots, f_{n-1}\right)$ with the inner product

$$
\langle\mathbf{f}, \mathbf{g}\rangle_{\mathcal{H}}=\sum_{0 \leq k \leq n-1}\left\langle f_{k}, g_{k}\right\rangle_{L^{2}\left(\mathbb{R}^{d}\right)}
$$

Observe that

$$
\mathcal{H} \otimes \mathcal{H}=L^{2}\left(\mathbb{R}^{2 d}\right)
$$

The super Gabor transform of a function $\mathbf{f}$ with respect to the "window" $\mathbf{g}=\left(g_{0}, \ldots g_{n-1}\right)$ such that

$$
\left\langle g_{i}, g_{j}\right\rangle_{L^{2}\left(\mathbb{R}^{d}\right)}=\delta_{i, j}
$$

is defined, for every $x, \omega \in \mathbb{R}^{d}$, as

$$
\mathbf{V}_{\mathbf{g}} \mathbf{f}(x, \omega)=\left\langle\mathbf{f}, M_{\omega} T_{x} \mathbf{g}\right\rangle_{\mathcal{H}} .
$$


That is to say,

This defines a map

$$
\mathbf{V}_{\mathbf{g}} \mathbf{f}(x, \omega)=\sum_{0 \leq k \leq n-1} V_{g_{k}} f_{k}(x, \omega)
$$

$$
\mathbf{V}_{\mathbf{g}} \mathbf{f}: \mathcal{H} \rightarrow L^{2}\left(\mathbb{R}^{2 d}\right)
$$

Since the vector $\mathbf{g}$ is extracted from an orthonormal sequence, most of the properties of the scalar Gabor transform are kept. In particular, the isometric property and orthogonality relations, extend to the vector valued case [1].

Proposition 1. Let $\mathbf{f}_{1}, \mathbf{f}_{2}, \in \mathcal{H}$. Then $\mathbf{V}_{\mathbf{g}} \mathbf{f}_{1}, \mathbf{V}_{\mathbf{g}} \mathbf{f}_{2} \in L^{2}\left(\mathbb{R}^{2 d}\right)$ and

$$
\left\langle\mathbf{V}_{\mathbf{g}} \mathbf{f}_{1}, \mathbf{V}_{\mathbf{g}} \mathbf{f}_{2}\right\rangle_{L^{2}\left(\mathbb{R}^{2 d}\right)}=\left\langle\mathbf{f}_{1}, \mathbf{f}_{2}\right\rangle_{\mathcal{H}}
$$

Therefore, $\mathbf{V}_{\mathbf{g}} \mathbf{f}$ is an isometry between Hilbert spaces.

Proof: First observe that, from (3) and (14),

$$
\left\langle V_{g_{k}} f_{k}, V_{g_{j}} f_{j}\right\rangle_{L^{2}\left(\mathbb{R}^{2 d}\right)}=\left\langle f_{k}, f_{j}\right\rangle_{L^{2}\left(\mathbb{R}^{d}\right)} \overline{\left\langle g_{k}, g_{j}\right\rangle_{L^{2}\left(\mathbb{R}^{d}\right)}}=\delta_{i, j} .
$$

Then, using (14) and (3),

$$
\begin{aligned}
\left\langle\mathbf{V}_{\mathbf{g}} \mathbf{f}_{1}, \mathbf{V}_{\mathbf{g}} \mathbf{f}_{2}\right\rangle_{L^{2}\left(\mathbb{R}^{2 d}\right)} & =\sum_{0 \leq k \leq n-1}\left\langle V_{g_{k}} f_{k}, V_{g_{k}} f_{k}\right\rangle_{L^{2}\left(\mathbb{R}^{d}\right)} \\
& =\sum_{0 \leq k \leq n-1}\left\langle f_{1, k}, f_{2, k}\right\rangle_{L^{2}\left(\mathbb{R}^{2 d}\right)} \\
& =\left\langle\mathbf{f}_{1}, \mathbf{f}_{2}\right\rangle_{\mathcal{H}}
\end{aligned}
$$

Of course,

$$
\left\|\mathbf{V}_{g} \mathbf{f}\right\|_{L^{2}\left(\mathbb{R}^{2 d}\right)}=\|\mathbf{f}\|_{\mathcal{H}}
$$

Now let $\mathbf{G}_{\mathrm{g}}$ stand for the subspace of $L^{2}\left(\mathbb{R}^{2 d}\right)$ constituted by the image of $\mathcal{H}$ under the vector valued Gabor transform $\mathbf{V}_{\mathbf{g}} \mathbf{f}$. We will call this the Gabor super space associated with the window $\mathbf{g}$ :

$$
\mathbf{G}_{\mathbf{g}}=\left\{\mathbf{V}_{\mathbf{g}} \mathbf{f}: \mathbf{f} \in \mathcal{H}\right\}
$$

Since

$$
\mathbf{V}_{\mathbf{g}} \mathbf{f}=\sum_{0 \leq k \leq n-1} V_{g_{k}} f_{k}
$$

and

$$
\left\langle V_{g_{k}} f_{k}, V_{g_{j}} f_{j}\right\rangle_{L^{2}\left(\mathbb{R}^{2 d}\right)}=\delta_{i, j}
$$


we know that every $F \in \mathbf{G}_{\mathbf{g}}$ can be written in a unique way in the form

$$
F=F_{0}+\ldots+F_{n-1}, \quad F_{k} \in \mathcal{G}_{g_{k}}
$$

That is to say,

$$
\mathbf{G}_{\mathrm{g}}=\mathcal{G}_{g_{0}} \oplus \ldots \oplus \mathcal{G}_{g_{n-1}}
$$

In the next proposition, as well as in many other places along the text, we identify $(x, \omega)$ with $z=x+i \omega$.

Proposition 2. The space $\mathbf{G}_{\mathbf{g}}$ is a Hilbert space with reproducing kernel given by

$$
\mathbf{k}(z, w)=\left\langle M_{\omega} T_{x} \mathbf{g}, M_{\eta} T_{u} \mathbf{g}\right\rangle_{\mathcal{H}}
$$

Proof: Let $\mathbf{F} \in \mathbf{G}_{\mathbf{g}}$. There exists $\mathbf{f} \in L^{2}\left(\mathbb{R}^{2 d}\right)$ such that $\mathbf{F}=\mathbf{V}_{\mathbf{g}} \mathbf{f}$. By definition, $\mathbf{k}(z,)=.\mathbf{V}_{\mathbf{g}} \mathbf{f}\left(\mathbf{M}_{\omega} \mathbf{T}_{\mathbf{x}} \mathbf{g}\right)$. Thus, using (16),

$$
\begin{aligned}
\langle\mathbf{F}, \mathbf{k}(z, .)\rangle_{L^{2}\left(\mathbb{R}^{2 d}\right)} & =\left\langle\mathbf{V}_{\mathbf{g}} \mathbf{f}, \mathbf{V}_{g} \mathbf{f}\left(\mathbf{M}_{\omega} \mathbf{T}_{\mathbf{x}} \mathbf{g}\right)\right\rangle_{L^{2}\left(\mathbb{R}^{2 d}\right)} \\
& =\left\langle\mathbf{f}, \mathbf{M}_{\omega} \mathbf{T}_{\mathbf{x}} \mathbf{g}\right\rangle_{\mathcal{H}} \\
& =\mathbf{V}_{\mathbf{g}} \mathbf{f}(\mathbf{z}) \\
& =\mathbf{F}(z) .
\end{aligned}
$$

3.3. Structure of Gabor and super Gabor spaces. In this section we provide an operator description of the Gabor and super Gabor spaces, showing, in the sense of [13],[14], [10], [11], how much room do such spaces occupy inside $L^{2}\left(\mathbb{R}^{2 d}\right)$.

For convenience write $L^{2}\left(\mathbb{R}^{2 d}\right)=L^{2}\left(\mathbb{R}^{d}\right) \otimes L^{2}\left(\mathbb{R}^{d}\right)$. Then define operators $U_{1}$ and $U_{2}$,

$$
U_{1,2}: L^{2}\left(\mathbb{R}^{d}\right) \otimes L^{2}\left(\mathbb{R}^{d}\right) \rightarrow L^{2}\left(\mathbb{R}^{d}\right) \otimes L^{2}\left(\mathbb{R}^{d}\right)
$$

with different actions on each variable in $\mathbb{R}^{d}: U_{1}(F)(x, \omega)=\left(\mathcal{F}^{-1} \otimes I\right)(F)(x, \omega)$ and $U_{2}(F)(x, \omega)=F(-x+\omega, \omega)$. Both operators are clearly unitary. In the proof of the next result we will use the involution operator * defined as

$$
g^{*}(x)=\overline{g(-x)}
$$

The next proposition is the analogue of Theorem 2.1 in [10]. It is essentially the inverse of the representation of the Gabor transform given in lemma 3.1.2 in $[7]$. 
Proposition 3. The operator $U=U_{2} U_{1}$ gives an isometrical isomorphism of the space $L^{2}\left(\mathbb{R}^{2 d}\right)$ into itself, under which the space of Gabor transforms $\mathcal{G}_{g}$ is mapped onto $L^{2}\left(\mathbb{R}^{d}\right) \otimes \mathcal{L}$, where $\mathcal{L}$ is the one dimensional subspace of $L^{2}\left(\mathbb{R}^{d}\right)$ generated by $\bar{g}$.

Proof: The operator $U=U_{2} U_{1}$ inherits the unitarity and linearity properties from $U_{2}$ and $U_{1}$. Using the notation (21) for the involution operator, we can write the Gabor transform (1) as the convolution product

$$
V_{g} f(x, \omega)=\left(\mathcal{F} f * M_{-x}(\mathcal{F} g)^{*}\right)(\omega) .
$$

Now, apply the operator $U_{1}$ to a general element of $\mathcal{G}_{g}$ written in the form (22). A calculation shows that $\mathcal{F}^{-1}\left((\mathcal{F} g)^{*}\right)=\bar{g}$. Combining this with the identity (4) and taking into account that $\mathcal{F}^{-1}\left[L^{2}\left(\mathbb{R}^{d}\right)\right]=L^{2}\left(\mathbb{R}^{d}\right)$, we see immediately that the image of the space $G_{g}$ under $U_{1}$ is the set of all functions in $L^{2}\left(\mathbb{R}^{2 d}\right)$ that can be written in the form $F(x, \omega)=f(\omega) \overline{g(\omega-x)}$. Applying $U_{2}$, we see that the image of the space $\mathcal{G}_{g}$ under $U$ is constituted by the functions of the form $F(x, \omega)=f(\omega) \overline{g(x)}$.

Observe that

$$
U\left[V_{g} f(x, \omega)\right]=f(\omega) \overline{g(x)}
$$

The result for super Gabor spaces follows now easily.

Theorem 1. The operator $U=U_{2} U_{1}$ gives an isometrical isomorphism of the space $L^{2}\left(\mathbb{R}^{2 d}\right)$ into itself, under which the space of super Gabor transforms $\mathbf{G}_{g}$ is mapped onto $\mathcal{H} \otimes \mathbf{L}$, where $\mathbf{L}$ is the subspace of $\mathcal{H}$ generated by $\mathbf{\mathbf { g }}$.

Proof: From the linearity of $U$ and (23),

$$
\begin{aligned}
U\left[\mathbf{V}_{\mathbf{g}} \mathbf{f}(x, \omega)\right] & =\sum_{0 \leq k \leq n-1} U\left[V_{g_{k}} f_{k}(x, \omega)\right] \\
& =\sum_{0 \leq k \leq n-1} f_{k}(\omega) \overline{g_{k}}(x) . \\
& =\langle\mathbf{f}, \mathbf{g}\rangle_{\mathcal{H}} .
\end{aligned}
$$

\section{Gabor and super Gabor spaces with Hermite windows}

4.1. Structure. We will use the following notation for Gabor spaces with Hermite windows.

$$
\mathcal{G}^{n}=\left\{V_{\Phi_{n}} f(x, \omega): f \in L^{2}\left(\mathbb{R}^{d}\right)\right\}
$$


We can apply Proposition 3 to the spaces $\mathcal{G}^{n}\left(\mathbb{R}^{d}\right)$.

Corollary 1. The operator $U$ gives an isometrical isomorphism of the space $L^{2}\left(\mathbb{R}^{2 d}\right)$ into itself, under which the Gabor spaces with Hermite window $\mathcal{G}^{n}\left(\mathbb{R}^{d}\right)$ is mapped onto $L^{2}\left(\mathbb{R}^{d}\right) \otimes \mathcal{L}^{n}$, where $\mathcal{L}^{n}$ is the one dimensional subspace of $L^{2}\left(\mathbb{R}^{d}\right)$ generated by $\overline{\Phi_{n}(x)}$.

Therefore, the image of the space $\mathcal{G}^{n}$ under $U$ is constituted by the functions of the form

$$
F(x, \omega)=f(\omega) \overline{\Phi_{n}(x)},
$$

where $f \in L^{2}\left(\mathbb{R}^{d}\right)$.

The vector valued version is

Corollary 2. The operator $U$ gives an isometrical isomorphism of the space $L^{2}\left(\mathbb{R}^{2 d}\right)$ into itself, under which the Gabor spaces with Hermite window $\mathcal{G}^{n}$ is mapped onto $\mathcal{H} \otimes \mathbf{L}^{\mathbf{n}}$, where $\mathbf{L}^{\mathbf{n}}$ is the subspace of $\mathcal{H}$ generated by $\boldsymbol{\Phi}_{n-1}=$ $\left(\overline{\Phi_{0}}, \ldots, \overline{\left.\Phi_{n-1}\right)}\right.$.

4.2. Reproducing kernel. Define the operator $\beta_{\zeta}$ on $\mathcal{F}\left(\mathbb{C}^{d}\right)$ by

$$
\beta_{\zeta} F(z)=e^{i \pi x \omega-\pi \frac{|\zeta|^{2}}{2}} e^{\pi \bar{\zeta} z} F(z-\zeta) .
$$

The operator $\beta_{\zeta}$ satisfies the intertwining property

$$
\beta_{\zeta} B=B M_{\omega} T_{x}, \quad z=x+i \omega .
$$

Theorem 2. The reproducing kernel of $\mathcal{G}^{n}$ is given by

$$
k^{n}(z, w)=\frac{1}{n !} e^{i \pi(x \omega-u \eta)+\frac{\pi}{2}\left(|w|^{2}-|z|^{2}\right)}\left(\frac{d}{d w}\right)^{n}\left[e^{\pi \bar{z} w-\pi|w|^{2}}(w-z)^{n}\right]
$$

Proof: Using (10) and (25), we obtain

$$
\begin{aligned}
k^{n}(z, w) & =\left\langle M_{\omega} T_{x} \Phi_{n}(x), M_{\eta} T_{u} \Phi_{n}(x)\right\rangle_{L^{2}\left(\mathbb{R}^{d}\right)} \\
& =\left\langle\beta_{z} B \Phi_{n}(x), \beta_{w} B \Phi_{n}(x)\right\rangle_{\mathcal{F}\left(\mathbb{C}^{d}\right)} \\
& =\frac{1}{n !} \pi^{n} e^{i \pi(x \omega-u \eta)-\frac{\pi}{2}\left(|z|^{2}+|w|^{2}\right)}\left\langle e^{\pi \bar{z} \zeta}(\zeta-z)^{n}, e^{\pi \bar{w} \zeta}(\zeta-w)^{n}\right\rangle_{\mathcal{F}\left(\mathbb{C}^{d}\right)} .
\end{aligned}
$$

Now, diferentiating the reproducing kernel equation $F(w)=\left\langle F(\zeta), e^{\pi \bar{w} \zeta}\right\rangle_{\mathcal{F}(\mathbb{C})}$, gives

$$
\left\langle F(\zeta), \zeta^{n-k} e^{\pi \bar{w} \zeta}\right\rangle_{\mathcal{F}\left(\mathbb{C}^{d}\right)}=\pi^{k-n} F^{(n-k)}(w)
$$


Therefore,

$$
\begin{aligned}
k^{n}(z, w) & =\frac{1}{n !} e^{i \pi(x \omega-u \eta)-\frac{\pi}{2}\left(|z|^{2}+|w|^{2}\right)} \sum_{0 \leq k \leq n-1}\left(\begin{array}{l}
n \\
k
\end{array}\right)(-\bar{w})^{k}\left\langle e^{\pi \bar{z} \zeta}(\zeta-z)^{n}, \zeta^{n-k} e^{\pi \bar{w} \zeta}\right\rangle \\
& =\frac{1}{n !} e^{i \pi(x \omega-u \eta)-\frac{\pi}{2}\left(|z|^{2}+|w|^{2}\right)} \sum_{0 \leq k \leq n-1}\left(\begin{array}{l}
n \\
k
\end{array}\right)(-\bar{w} \pi)^{k}\left(\frac{d}{d w}\right)^{n-k}\left[e^{\pi \bar{z} w}(w-z)^{n}\right] \\
& =\frac{1}{n !} e^{i \pi(x \omega-u \eta)-\frac{\pi}{2}\left(|z|^{2}+|w|^{2}\right)} e^{\pi|w|^{2}}\left(\frac{d}{d w}\right)^{n}\left[e^{-\pi|w|^{2}} e^{\pi \bar{z} w}(w-z)^{n}\right] .
\end{aligned}
$$

and the result follows.

Corollary 3. The functions in $\mathcal{G}^{n}$ satisfy the growth estimate

$$
|f| \leq \frac{1}{\sqrt{n !}}\|f\|_{\mathcal{G}^{(n)}\left(\mathbb{R}^{d}\right)}
$$

Proof: From the representation of the reproducing kernel (26)

$$
\left\|k^{n}(., z)\right\|_{\mathcal{G}^{(n)}}=\sqrt{k^{n}(z, z)}=\frac{1}{\sqrt{n !}} .
$$

and use (12).

Corollary 4. The orthogonal projection of $L^{2}\left(\mathbb{R}^{2}\right)$ over the space $\mathcal{G}^{n}$ is given by the operator

$$
\left(p^{n} F\right)(w)=\int_{L^{2}\left(\mathbb{R}^{2}\right)} f(z) \overline{k^{n}(w, z)} d z
$$

Proof: This follows at once from the fact that $k^{n}(w, z)$ is the reproducing kernel of $\mathcal{G}^{n}$.

Remark 1. There are other ways of computing the reproducing kernel, for instance, in the form of an double infinite sum, by using the series development of the Hermite functions. We choose the above form because it becomes easy to evaluate the diagonal required in corollary 5.

From (20) we see that the reproducing kernel of $\mathbf{G}^{n}$ is given as

$$
\mathbf{k}^{n}(z, w)=\sum_{0 \leq k \leq n-1} \frac{e^{i \pi(x \omega-u \eta)+\frac{\pi}{2}\left(|w|^{2}-|z|^{2}\right)}}{k !}\left(\frac{d}{d w}\right)^{k}\left[e^{\pi \bar{z} w-\pi|w|^{2}}(w-z)^{k}\right]
$$




\section{Polyanalytic Fock spaces}

5.1. Definitions. We will use multi-index notation in such a way that there will be little difference between the one and the $d$-dimensional case.

The Fock space of polyanalytic functions $[4], \mathbf{F}^{n}\left(\mathbb{C}^{d}\right)$, consists on all functions satisfying the equation

$$
\left(\frac{d}{d \bar{z}}\right)^{n} F(z)=0
$$

and such that

$$
\int_{\mathbb{C}^{d}}|F(z)|^{2} e^{-\pi|z|^{2}} d z<\infty .
$$

Functions satisfying (28) are known as polyanalytic functions of order $n$. Since (28) generalizes the Cauchy-Riemann equation

$$
\frac{d}{d \bar{z}} F(z)=0
$$

then the space $\mathbf{F}^{n}\left(\mathbb{C}^{d}\right)$ is a generalization of the Bargmann-Fock space of analytic functions, $\mathcal{F}(\mathbb{C})=\mathbf{F}^{1}\left(\mathbb{C}^{d}\right)$, with the same inner product,

$$
\langle F, G\rangle_{L^{2}\left(\mathbb{C}^{d}, e^{\left.-\pi|z|^{2}\right)}\right.}=\int_{\mathbb{C}^{d}} F(z) \overline{G(z)} e^{-\pi|z|^{2}} d z .
$$

Observe also that this implies

$$
\langle F, G\rangle_{\mathbf{F}^{n}\left(\mathbb{C}^{d}\right)}=\left\langle e^{\pi \frac{|z|^{2}}{2}} F, e^{\pi \frac{|z|^{2}}{2}} G\right\rangle_{L^{2}\left(\mathbb{R}^{2 d}\right)} .
$$

We will need some results from [1].

Proposition A. The set of functions $\left\{e_{k, m}\right\}_{0 \leq k \leq n-1 ; \ldots m \geq 0}$, where

$$
e_{k, m}(z)=\left(\pi^{|k|} k !\right)^{-\frac{1}{2}} e^{\pi|z|^{2}}\left(\frac{d}{d z}\right)^{k}\left[e^{-\pi|z|^{2}} e_{m}(z)\right]
$$

is an orthogonal basis of $\mathbf{F}^{n}\left(\mathbb{C}^{d}\right)$.

Definition 1. The true polyanalytic Fock space of order $n$ is defined as

$$
\mathcal{F}^{n}\left(\mathbb{C}^{d}\right)=\operatorname{Span}\left[\left\{e_{n, m}(z)\right\}_{m \geq 0}\right] .
$$

The next proposition connects Gabor transforms with Hermite windows with polyanalytic Bargmann transforms. For the proof see [1]. 
Proposition B. The true polyanalytic Bargmann transform, defined by the formula

$$
\left(\mathcal{B}^{n} f\right)(z)=\left(\pi^{|n|} n !\right)^{-\frac{1}{2}} e^{\pi|z|^{2}}\left(\frac{d}{d z}\right)^{n}\left[e^{-\pi|z|^{2}} F(z)\right],
$$

where $F(z)=(B f)(z)$ is a polyanalytic function of order $n+1$ on $\mathbb{C}^{d}$. This transform is an isometric isomorphism

$$
\mathcal{B}^{n}: L^{2}\left(\mathbb{R}^{d}\right) \rightarrow \mathcal{F}^{n}\left(\mathbb{C}^{d}\right),
$$

and if we write $z=x+i \omega$, it is related to the Gabor transform with Hermite windows as follows:

$$
V_{\Phi_{n}} f(x, \omega)=e^{i \pi x \omega-\pi \frac{|z|^{2}}{2}}\left(\mathcal{B}^{n} f\right)(z)
$$

5.2. The multiplier isomorphism. Consider the operator $E$ such that

$$
E: f \rightarrow M f
$$

where

$$
M(z)=e^{\pi \frac{|z|^{2}}{2}-i \pi x \omega}
$$

with $z=x+i \omega$.

Proposition 4. E is an isometric isomorphism,

$$
E: \mathcal{G}^{n}\left(\mathbb{C}^{d}\right) \rightarrow \mathcal{F}^{n}\left(\mathbb{C}^{d}\right)
$$

and

$$
E: \mathbf{G}^{n}\left(\mathbb{C}^{d}\right) \rightarrow \mathbf{F}^{n}\left(\mathbb{C}^{d}\right)
$$

Proof: Clearly, E is isometric. From (9) and (30) one can easily see that

$$
e_{k, m}(z)=\left(\mathcal{B}^{k} \Phi_{m}\right)(z)
$$

Since $\left\{\Phi_{m}\right\}_{m \geq 0}$ is a basis of $L^{2}\left(\mathbb{R}^{d}\right)$, then $\left\{V_{\Phi_{n}} \Phi_{m}\right\}_{m \geq 0}$ is a basis of $\mathcal{G}^{n}$. Since

$$
E\left(V_{\Phi_{n}} \Phi_{m}\right)=e^{\pi \frac{|z|^{2}}{2}-i \pi x \omega} V_{\Phi_{n}} \Phi_{m}=\mathcal{B}^{n}\left(\Phi_{m}\right)=e_{n, m}(z),
$$

then $E$ is a unitary isomorphism $\mathcal{G}^{n} \rightarrow \mathcal{F}^{n}$, since $\left\{e_{n, m}(z)\right\}_{m \geq 0}$ is an orthogonal basis of $\mathcal{F}^{n}\left(\mathbb{C}^{d}\right)$. Now, applying (19) to $\mathbf{G}^{n}$, we have the decomposition:

$$
\mathrm{G}^{n}=\mathcal{G}^{0} \oplus \ldots \oplus \mathcal{G}^{n-1} \text {. }
$$


On the other side, from proposition A and the definition of $\mathcal{F}^{n}\left(\mathbb{C}^{d}\right)$, it is clear that

$$
\mathbf{F}^{n}\left(\mathbb{C}^{d}\right)=\mathcal{F}^{0}\left(\mathbb{C}^{d}\right) \oplus \ldots \oplus \mathcal{F}^{n-1}\left(\mathbb{C}^{d}\right) .
$$

Therefore, we also have an unitary isomorphism $\mathbf{G}^{n} \rightarrow \mathbf{F}^{n}\left(\mathbb{C}^{d}\right)$.

This proposition allows the transference of results from $\mathcal{G}^{n}$ to $\mathcal{F}^{n}\left(\mathbb{C}^{d}\right)$ and from $\mathbf{G}^{n}$ to $\mathbf{F}^{n}\left(\mathbb{C}^{d}\right)$.

5.3. Structure. Now, the operator $U^{*}=E^{-1} U$ gives an isometrical isomorphism

$$
U^{*}: L^{2}\left(\mathbb{C}^{d}, e^{-\pi|z|^{2}}\right) \rightarrow L^{2}\left(\mathbb{C}^{d}, e^{-\pi|z|^{2}}\right)
$$

Thus we recover the results of theorem 2.1 and corollary 2.2 in [13].

Theorem 3. The operator $U^{*}=E^{-1} U$ maps the true polyanalytic Fock space $\mathcal{F}^{n}\left(\mathbb{C}^{d}\right)$ onto $L^{2}\left(\mathbb{R}^{d}\right) \otimes \mathcal{L}$, where $\mathcal{L}$ is the one dimensional subspace of $L^{2}\left(\mathbb{R}^{d}\right)$ generated by $\overline{\Phi_{n}(x)}$.

Proof: Since $E$ is unitary, given a function $F \in \mathcal{F}^{n}\left(\mathbb{C}^{d}\right)$, we know that $E^{-1} F \in \mathcal{G}^{n}$. By corollary 1, we know that $\left(U E^{-1} F\right)(x, w)=f(w) \overline{\Phi_{m}}(x)$ and the result follows.

In a similar way, the next theorem follows from corollary 2 .

Theorem 4. The polyanalytic Fock space $\mathbf{F}^{n}\left(\mathbb{C}^{d}\right)$ is mapped by $U^{*}$ onto $L^{2}\left(\mathbb{R}^{d}\right) \otimes \mathbf{L}$, where $L$ is the subspace of $L^{2}\left(\mathbb{R}^{d}\right)$ generated by $\boldsymbol{\Phi}_{n-1}=\left(\overline{\Phi_{0}}, \ldots, \overline{\Phi_{n-1}}\right)$.

5.4. The reproducing kernel. As a further application of the multiplier isomorphism $E$, we show that the space $\mathcal{F}^{n}\left(\mathbb{C}^{d}\right)$ has a reproducing kernel and compute it explicitly. We first relate it to the reproducing kernel of the Gabor spaces with Hermite windows, providing a generalization of (11) (the case $n=0)$.

Proposition 5. Let $k^{n}(z, w)$ be the reproducing kernel of the space $\mathcal{G}^{n}$. The space $\mathcal{F}^{n}(\mathbb{C})$ is a Hilbert space with a reproducing kernel, $K^{n}(z, w)$ satisfying

$$
K^{n}(z, w)=e^{i \pi(u \eta-x \omega)+\pi \frac{|z|^{2}+|w|^{2}}{2}} k^{n}(z, w) .
$$

Proof: Given $F \in \mathcal{F}^{n}\left(\mathbb{C}^{d}\right)$, there exists $f \in \mathcal{G}^{n}$ such that $E f=F$, that is, $f(z)=e^{i \pi x \omega-\pi \frac{|z|^{2}}{2}} F(z)$. Since $\mathcal{G}^{n}$ is a Hilbert space with reproducing kernel $k^{n}(z, w)$, the reproducing property gives

$$
f(z)=\left\langle f(w), k^{n}(z, w)\right\rangle_{\mathcal{G}^{n}}
$$


or

$$
\begin{aligned}
e^{i \pi x \omega-\pi \frac{|z|^{2}}{2}} F(z) & =\left\langle e^{i \pi u \eta-\pi \frac{|w|^{2}}{2}} F(w), k^{n}(z, w)\right\rangle_{\mathcal{G}^{n}} \\
& =\left\langle e^{i \pi u \eta+\pi \frac{|w|^{2}}{2}} F(w), k^{n}(z, w)\right\rangle_{\mathcal{F}^{n}\left(\mathbb{C}^{d}\right)} .
\end{aligned}
$$

Therefore,

$$
F(z)=\left\langle F(w), e^{i \pi(u \eta-x \omega)+\pi \frac{|z|^{2}+|w|^{2}}{2}} k^{n}(z, w)\right\rangle_{\mathcal{F}^{n}\left(\mathbb{C}^{d}\right)}=\left\langle F(w), K^{n}(z, w)\right\rangle_{\mathcal{F}^{n}\left(\mathbb{C}^{d}\right)}
$$

and $K^{n}(z, w)$ is the reproducing kernel of $\mathcal{F}^{n}\left(\mathbb{C}^{d}\right)$.

Combining (35) and (26) gives:

Corollary 5. The reproducing kernel of $\mathcal{F}^{n}\left(\mathbb{C}^{d}\right)$ is given by

$$
K^{n}(z, w)=\frac{1}{n !} e^{\pi|w|^{2}}\left(\frac{d}{d w}\right)^{n}\left[e^{\pi \bar{z} w-\pi|w|^{2}}(w-z)^{n}\right]
$$

Again, from the reproducing kernel we obtain an estimate for the modulus of functions in $\mathcal{F}^{n}\left(\mathbb{C}^{d}\right)$.

Corollary 6. The functions in $\mathcal{F}^{n}\left(\mathbb{C}^{d}\right)$ satisfy

$$
|F| \leq \frac{1}{n !}\|F\|_{\mathcal{F}^{n}\left(\mathbb{C}^{d}\right)} e^{\frac{\pi}{2}|w|^{2}}
$$

Proof: Since the norm of the reproducing kernel is

$$
\left\|K^{n}\right\|_{\mathcal{F}^{n}\left(\mathbb{C}^{d}\right)}=\sqrt{K^{n}(z, z)}=\frac{e^{\frac{\pi}{2}|w|^{2}}}{n !} .
$$

the estimate follows at once from (12)

Another immediate consequence of the reproducing kernel is the orthogonal projection over the true polyanalytic Fock spaces.

Corollary 7. The orthogonal projection of $L^{2}\left(\mathbb{C}^{d}, e^{-\pi|z|^{2}}\right)$ over the space $\mathcal{F}^{n}\left(\mathbb{C}^{d}\right)$ is given by the operator

$$
\left(P^{n} F\right)(w)=\int_{\mathbb{C}^{d}} F(z) K^{n}(w, z) e^{-\pi|z|^{2}} d z .
$$

Proof: This follows immediately from the fact that $K^{n}(w, z)$ is the reproducing kernel of $\mathcal{F}^{n}\left(\mathbb{C}^{d}\right)$. 
We also see that the reproducing kernel of $\mathbf{G}^{n}$ is given as

$$
\mathbf{K}^{n}(z, w)=\sum_{0 \leq k \leq n-1} \frac{e^{\pi|w|^{2}}}{k !}\left(\frac{d}{d w}\right)^{k}\left[e^{\pi \bar{z} w-\pi|w|^{2}}(w-z)^{k}\right]
$$

and that functions in $\mathbf{G}^{n}$ satisfy

$$
|\mathbf{F}| \leq\left(\sum_{0 \leq k \leq n-1} \frac{1}{k !}\right)^{1 / 2}\|\mathbf{F}\|_{\mathcal{F}^{n}\left(\mathbb{C}^{d}\right)} e^{\frac{\pi}{2}|w|^{2}}
$$

Remark 2. The corresponding estimates for the growth of functions in polyanalytic Bergman spaces in the unit disk have been obtained in [12], using direct computations and combinatorial arguments.

Remark 3. An alternative form of the reproducing kernel can be obtained from the fact that, since $\left\{e_{n, m}(z)\right\}_{m=0,1, \ldots}$ is a basis of $\mathcal{F}^{n}\left(\mathbb{C}^{d}\right)$,

$$
K^{n}(z, w)=\sum_{m=0}^{\infty} e_{n, m}(z) \overline{e_{n, m}(w)} .
$$

This gives the curious identity

$\left(\frac{d}{d w}\right)^{n}\left[e^{\pi \bar{z} w-\pi|w|^{2}}(w-z)^{n}\right]=\sum_{m=0}^{\infty} \frac{e^{\pi|z|^{2}}}{\pi^{n}} \frac{d^{n}}{d z^{n}}\left[e^{-\pi|z|^{2}} e_{m}(z)\right] \frac{d^{n}}{d \bar{w}^{n}}\left[e^{-\pi|w|^{2}} \overline{e_{m}(z)}\right]$.

\section{References}

[1] L. D. Abreu, Sampling and interpolation in the Bargmann-Fock space of polyanalytic functions, under review, http://arxiv.org/abs/0901.4386v4.

[2] G. Ascensi, J. Bruna, Model space results for the Gabor and Wavelet transforms, IEEE Trans. Inf. Theor., to appear, http://arxiv.org/abs/0804.3254.

[3] R. Balan, Multiplexing of signals using superframes, In SPIE Wavelets applications, volume 4119 of Signal and Image processing XIII, pag. 118-129 (2000).

[4] M. B. Balk, Polyanalytic functions, Akad. Verlag, Berlin (1991).

[5] I. Daubechies, "Ten lectures on wavelets", CBMS-NSF Regional conference series in applied mathematics (1992).

[6] H. Führ, Simultaneous estimates for vector-valued Gabor frames of Hermite functions. Adv. Comput. Math. 29 , no. 4, 357-373, (2008).

[7] K. Gröchenig, "Foundations of time-frequency analysis", Birkhäuser, Boston, (2001).

[8] K. Gröchenig, Y. Lyubarskii, Gabor frames with Hermite functions, C. R. Acad. Sci. Paris, Ser. I 344 157-162 (2007).

[9] K. Gröchenig, Y. Lyubarskii, Gabor (Super)Frames with Hermite Functions, preprint, arXiv:0804.4613.

[10] O. Hutnik, On the structure of the space of wavelet transforms, C. R. Math. Acad. Sci. Paris 346 , no. 11-12, 649-652, (2008). 
[11] O. Hutnik, A note on wavelet subspaces, Monatsh. Math, online published.

[12] A. K. Ramazanov, Representation of the space of polyanalytic functions as the direct sum of orthogonal subspaces. Application to rational approximations. (Russian) Mat. Zametki 66 (1999), no. 5, 741-759; translation in Math. Notes 66 (1999), no. 5-6, 613-627 (2000).

[13] N. M. Vasilesvski, Poly-Fock spaces, Differential operators and related topics, Vol. I (Odessa, 1997), 371-386, Oper. Theory Adv. Appl., 117, Birkhäuser, Basel, (2000).

[14] N. M. Vasilesvski, On the structure of Bergman and poly-Bergman spaces. Integral Equations Operator Theory 33, no. 4, 471-488, (1999).

Luis DANiEl ABreu

CMUC, Department of Mathematics, University of Coimbra, 3001-454 Coimbra, Portugal

E-mail address: daniel@mat.uc.pt 